\title{
Lesthète et le « mauvais garçon ». La correspondance entre William Ritter et Francis Carco (I9I4-I9I7)
}

\author{
Fabien Dubosson \\ Archives littéraires suisses
}

\begin{abstract}
En 19I4, William Ritter entame une correspondance avec Francis Carco, qui vient de connaître un succès de scandale avec son récit Jésus-la-Caille. Ritter est fasciné par ce roman, pour des raisons esthétiques, mais aussi plus intimes, que révèlent les lettres échangées avec son cadet de vingt ans. Quant à Carco, il profitera de ce rapport épistolaire pour affermir l'image auctoriale qu'il cherche désormais à se donner. L'article se propose donc de comprendre, d'une part, cet intérêt de Ritter pour un genre - le roman d'Apaches - fort éloigné de ses propres conceptions littéraires, et de l'autre, la poétique que Carco développe face à ce correspondant inattendu.
\end{abstract}

Keywords: William Ritter, Francis Carco, correspondance, roman, posture

C'est un soir de janvier I9I4 que William Ritter fait la connaissance de Jésus-la-Caille et de son créateur, « M. Pierre [sic] Carco », " connaissance qu['il] ne regrette pas », ainsi que nous l'apprend le journal de l'écrivain. Peu après les avoir découverts dans un numéro du Mercure de France, il entreprend en effet la rédaction d'un article conséquent sur ce court roman dont le sujet et l'esthétique l'ont durablement impressionné. Il est vrai que le thème en est peu commun, même pour un habitué des audaces décadentistes : dans une langue où alternent efficacement usage de l'argot et lyrisme d'une sobre élégance, Francis Carco y met en scène «la Caille», un jeune prostitué de Montmartre, et ses amours compliquées avec Bambou, petit voyou qui purge sa peine de prison à la Santé, la Puce - son frère cadet - et surtout Fernande, une prostituée que deux « affranchis » - le Corse et Pépéla-Vache - se disputent, mais qui succombe un temps aux charmes androgynes du héros éponyme. Les rebondissements dramatiques du récit sont surtout l'occasion pour Carco de dépeindre un milieu situé à la «marge » de la société - comme de la littérature - de l'époque, et dont les quartiers de prédilection sont alors Montmartre et Belleville : figures de la pègre, maquereaux, prostitués des deux sexes, ainsi que leurs clients - tous épiés et pourchassés par la « Rousse » et ses «bourdilles » (la police des mœurs et ses indicateurs). Mais c'est surtout son personnage principal qui fera date - les prostitués masculins ayant très rarement jusque-là donné leur nom à 
un roman, fût-il du genre " récit d'Apaches ». Jean Genet s'en souviendra d'ailleurs pour la figure de Divine dans Notre-Dame-des-Fleurs. Ritter, lui, ne tarit pas d'éloge sur ce texte, dès sa première lecture : "C'est d'une vie malgré le sujet sinistre ! Et les paysages brefs et bien vus ! Et puis... et puis... Faut-il avouer que [ce si joli] la Caille et [son] Bambou[, si expertement signalés, avec une certaine poésie même,] me touchent profondément ? Où le cœur va-t-il se nicher? Comme quoi les pires sacripans sont sauvés par un éclair de vraie passion... »".

Un compte rendu sera donc rédigé pour La Chronique politique, littéraire, économique de Marseille ${ }^{2}$. Bien qu'il s'agisse là d'un article parmi beaucoup d'autres dans la production de Ritter, il reste singulier par l'intérêt porté à un ouvrage dont l'esthétique semble, au premier abord, trancher avec ses propres conceptions littéraires. Il s'accompagne en outre - et c'est là le plus intéressant - d'un échange épistolaire entre l'écrivain neuchâtelois et l'auteur de Jésus-la-Caille. Dans le fonds conservé aux ALS, vingt-et-une lettres de Carco ont été répertoriées ${ }^{3}$, l'une d'elle acompagnée d'une photo, une autre d'un dessin de l'auteur le représentant de profil. Celles de Ritter sont présentes dans les monumentaux copies-lettres que l'écrivain tenait de manière systématique pour la plupart de ses envois ${ }^{4}$. Les premières lettres sont envoyées en février I9I4, et la correspondance s'achève, pour l'essentiel, en I9I7; elle est la plus abondante dans les premières années de la guerre, notamment en I9I5 et I9I6, au moment où Carco, devenu boulanger militaire à l'arrière, dans le cantonnement de Gray près de Besançon, attend son hypothétique affectation sur le front ; il intégrera finalement, en février i9ı6, grâce à l'intermédiaire de Jean Paulhan, une école de pilotes près de Dijon (Carco 2004 : XXXIV; Bedu 200I : I82).

Cette correpondance, initiée par Ritter, prolonge au départ l'enchantement de sa lecture de Jésus-la-Caille. Puis elle devient l'occasion d'une vé-

I «Journal ( (Munich, grand cahier noir), mardi 20 janvier 19I4, Fonds Ritter, Archives littéraires suisses (ALS), Berne. Tout au long de l'article, nous signalons entre crochets les ajouts autographes supralinéaires ou en marge, ainsi que certains éclaircissements de notre part ; nous conservons, pour toutes les citations, l'orthographe d'origine, ainsi que les guillemets anglais, etc.

2 Le manuscrit de ce compte rendu se trouve dans le Fonds Ritter des ALS, sous le titre : «Un roman d'aujourdhuy » [sic]. C'est ce texte - raturé, corrigé, avec de nombreux ajouts autographes, et vraisemblablement envoyé tel quel à la rédaction du journal, si l'on en juge par l'enveloppe qui l'accompagne - que nous citerons.

3 Le fonds étant encore en cours d'inventaire, il est fort probable que quelques lettres de Carco se trouvent toujours classées par date, sans distinction d'expéditeur, avec l'ensemble de la correspondance de l'écrivain neuchâtelois. L'inventaire de Josef Tcherv signale les dates extrêmes suivantes pour la correspondance avec Carco : I9I4-I943.

4 La lisibilité de ces missives recopiées et l'identification des destinataires étant souvent difficiles, nous ne pouvons avancer qu'avec prudence le nombre de lettres adressées à Carco : nous en avons dénombré une vingtaine pour les années qui nous ont intéressé. 
ritable projection des représentations du « mauvais garçon », telles que ce court roman les met en scène, sur la personne même de Carco qui, comme on va le voir, se prêtera d'assez bonne grâce à ce jeu de séduction épistolaire. Ritter n'aura vraisemblablement pas l'occasion de rencontrer Carco, dans ces années-là comme plus tard, et celui-ci restera avant tout, par les lettres comme par son récit, un interlocuteur « de papier », si l'on peut dire, objet de fantasmes et prétexte à l'aveu d'assez curieuses nostalgies.

Quant à Carco, ses lettres présentent un double intérêt. Elles valent d'abord comme un témoignage sur la guerre, vue pour l'essentiel de l'arrière et dans le sentiment de « cafard » qui s'attache à la vie d'un encaserné attendant son envoi sur le front. Carco, qui approche de la trentaine et qui n'est plus tout à fait un débutant des lettres - malgré ses protestations de modestie face à Ritter ${ }^{5}$ - livre en outre à son correspondant des vues essentielles sur ce que l'on pourrait appeler son art poétique, lequel se situe, apparemment, à l'opposé de celui défendu par l'auteur d'Ægyptiacque. Il se pourrait même que ce soit la confrontation avec la culture et le style très « artiste » de ce dernier qui ait aiguisé chez Carco le besoin de telles confidences sur son « métier » et sur ses ambitions littéraires.

Parcourir cet échange offre ainsi, d'une part, quelques vues inédites sur une tentative de "mentorat » à distance pour le moins surprenante chez Ritter, au vu de la personnalité du " protégé » qu'il s'est choisi - tentative restée d'ailleurs sans lendemain (contrairement au cas du Corbusier) ; de l'autre, des aperçus sur des conceptions divergentes de la littérature, dans un moment de basculement majeur des sensibilités (les années d'immédiat avant-guerre et de guerre), où les avatars du symbolisme font place à des courants artistiques nouveaux (en particulier les avant-gardes), plus attentifs aux arts «populaires » et « mineurs ». Pour modeste que nous paraisse aujourd'hui sa place dans l'histoire littéraire, Francis Carco, figure de la bohème montmartroise proche un temps des représentants de l'« esprit nouveau » (Apollinaire, Max Jacob, Modigliani...), aura participé lui aussi à ce mouvement général de redéfinition des sensibilités d'avant-guerre, avant de devenir une " personnalité » du monde littéraire des années 1920-30, notamment comme romancier à succès. Il n'est donc pas sans intérêt de nous attarder sur quelques aspects significatifs de cet échange épistolaire entre Ritter et Carco.

Revenons d'abord à l'expérience de lecture de Ritter, telle qu'elle apparaît dans son article pour La Chronique. Il s'y trouve précisément une attention à ce qui fait la modernité de Jésus-la-Caille. Certes, la défense de ce récit

5 Dans les années d'avant-guerre, Carco participe en effet, avec d'autres auteurs " provinciaux » - Paul-Jean Toulet, Claudien, Tristan Derème, Léon Vérane, Philippe Chabaneix, Jean Pellerin, Jean-Marc Bernard -, à ce courant de poètes qualifiés de «fantaisistes », qui publient un temps dans les mêmes revues (Décaudin 1982). 
au sujet « scabreux » (Julien Green, cité dans Carco 2004 : 6) va d'abord s'articuler sur le plan moral, avec un sous-texte apologétique sans doute en partie sincère, mais qui mobilise aussi les lieux communs rhétoriques du genre. Alors qu'il va attirer « un recri d'abomination et de surprise » ( Un roman d'aujourdhuy », p. I), le roman de Carco est en fait « un fort beau livre, pas du tout si immoral puisqu'il dégage de la terreur et une immense tristesse »; il irait même jusqu'à susciter « le désir fou d'une parole de salut, d'une prédication franciscaine » (ibid.). L'optique est ici clairement aurevillienne, et rejoint la préface, elle-même ambiguë, des Diaboliques : la peinture des « pires misérables » devrait susciter une «profonde pitié » chez les lecteurs ${ }^{6}$. Mais Ritter ne peut cacher ce qui le retient aussi dans ce texte, et qui contredit ce premier argument proposé en sa faveur et rappelé tout au long de l'article : il s'agit de sa séduction proprement érotique :

Rarement au reste la beauté physique [, l'attrait spécial,] de ces rôdeurs, filles ou bardaches, ont été appréciés et caractérisés avec une aussi experte clairvoyance. Aux lecteurs les plus éloignés d'y être initiés le charme malsain du ruisseau est rendu sensible (4).

Certes, cet attrait cherche encore à trouver sa légitimité par le biais d'autres références artistiques - dans celles, par exemple, qui ont illustré la sensibilité fin-de-siècle, comme Rops ou Steinlen :

Arsouilles, c'est vite dit, mais arsouilles dont la séduction n'est pas tue, dont le charme pervers est rendu sensible comme par le crayon ou le burin de quelques uns de ces artistes qui ont le sens de la beauté des gueux [et des corps dépravés], un Rops, un Steinlen, un Brangwyn (2-3).

Mais cette " beauté des gueux » sert ici un peu de paravent à ce qui constitue la réelle nouveauté thématique de ce roman, que Ritter se garde de relever explicitement, tout en le mettant indirectement en avant : sa teneur homoérotique - et même « polysexuelle ». Il reste toutefois que cet intérêt devient plus lisible dans d'autres références évoquées plus loin dans l'article : Le Portrait de Dorian Gray de Wilde et le Satiricon (4). Les oxymores utilisés pour qualifier les personnages de Carco tendent aussi à souligner cet

6 On peut comparer en effet cet argument avec la préface de Barbey d'Aurevilly : " [Les Diaboliques] ont pourtant été écrites par un moraliste chrétien, mais qui se pique d'observation vraie, quoique très hardie, et qui croit - c'est sa poétique, à lui - que les peintres puissants peuvent tout peindre et que leur peinture est toujours assez morale quand elle est tragique et qu'elle donne l'horreur des choses qu'elle retrace. Il n'y a d'immoral que les Impassibles et les Ricaneurs. Or, l'auteur de ceci, qui croit au Diable et à ses influences dans le monde, n'en rit pas, et il ne les raconte aux âmes pures que pour les en épouvanter » (Barbey d'Aurevilly 20I0: 83I). 
attrait paradoxal pour le lecteur : le «stigmate de leur vice » semble bien, en effet, la raison de leur « chic canaille » et de leur « beauté cruelle » (" peut-on pleinement mépriser un être beau » ?, remarque d'ailleurs Ritter à propos de La Caille). La distinction, faite dans l'article, entre différents lectorats auxquels s'adresserait le texte instaure de même des niveaux de lecture où la reconnaissance d'un désir érotique singulier pourrait valoir, précisément, comme ligne de partage.

L'autre force du récit de Carco, selon le critique, tiendrait dans sa capacité à allier l'audace du thème à celle des moyens. L'utilisation de l'argot en fait partie. Ritter y voit même un « tour de force » de l'écrivain, qui est parvenu à faire comprendre les dialogues au lecteur sans avoir donné " une seule explication de ces mots d'argot ». Il signale aussi la « pudeur » de ce langage, dont le goût pour « l'euphémisme abréviatif ou métaphorique » (3), qui remplace « l'expression criminelle » et la « description dégradante », n'est pas sans faire penser aux « termes nobles » de la tragédie classique (ibid.). Enfin, il y a le caractère proprement documentaire de tels usages linguistiques, qui pourront sans doute intéresser « quelque historien futur » " prenant la succession de M. Lazare Senean $»^{7}(5)$. Cette description d'un milieu, avec son sociolecte et ses habitudes, et menée avec une " effarante compétence » par Carco, donnerait donc, selon Ritter, une valeur « presque scientifique » à ce récit :

[...] ce qui [...] est [nouveau] c'est la rigueur presque scientifique de la démonstration, l'intensité de vie et d'évocation de ce monde sans nom, la sobriété de la mise en scène, l'absence de présentation et la plantation tout de gô, bien droites et viables, sur leurs pieds, de chaque personnage en deux ou trois traits au plus (2).

La méthode resterait toutefois bien distincte de celle de Zola - précisément par la "sobriété de la mise en scène » et par son sens de la poésie du paysage urbain ${ }^{8}$. Dans tous les cas, le naturalisme demeure pour Ritter, dans sa critique littéraire, le contre-exemple par excellence, par une forme de dédain envers Zola qu'il a gardé en partage avec les principaux représentants de la mouvance symboliste-décadente - et cela, même si Carco n'a jamais caché, de son côté, son admiration pour l'auteur de L'Assommoir.

En fait, contrairement à ses devanciers naturalistes, Jésus-la-Caille serait un « livre rapide et parfois cinématographique - moins [le tremblement, car il est au contraire d'une écriture résolue et ferme où rien ne trahit un dé-

7 Il s'agit du philologue français d'origine roumaine Lazar Sainéan, spécialiste notamment de l'argot et de la langue de Rabelais.

8 «Oh! Comme nous sommes loins [sic] des lourdauds et interminables panoramas de Paris de Zola et plus loin encore de [la] ridicule adoration de Paris de la Louise de Charpentier » (5). 
but] » (5). Par là, l'esthétique neuve de ce roman fait sentir ce qui se joue dans la « vie ardente » de l'homme moderne : vitesse, goût du sport, contraction du temps et de l'espace - le roman « moderniste » des années vingt, version Paul Morand, n'est pas loin :

Tout entier au reste cela se meut d'une esthétique nouvelle qui, elle aussi, participe à la trépidation imprimée à notre vie moderne par les sports, la fièvre de la vitesse, [la fièvre de dévorer le temps comme l'espace...] Et j'avoue avoir été secoué comme rarement par ces [quelques chapitres] brefs et outranciers avec simplicité (5).

Cette poésie violente et nouvelle, attachée à renouveler la perception des «bas-fonds » et des marges urbaines avec des moyens neufs, annonce une épopée encore à venir, à laquelle Carco pourra peut-être donner forme :

Et si je m'étonne qu'aucun vrai romancier ne se soit encore emparé des hauts faits de la bande Bonnot Garnier du moins est-il indéniable que ces Pépé la Vache et l'Espagnol [deux personnages d'« affranchis » dans le roman] portent-ils la marque de leur temps. Ceci n'est qu'une louche et trouble - et exquise idylle. M. Carco atteindra-t-il peut-être un de ces jours à l'épopée (3).

Les principaux points de cette analyse du roman de Carco vont être repris, par bribes, dans les lettres envoyées par Ritter au jeune auteur. La correspondance dévoile cependant les raisons personnelles - et implicites dans l'article - expliquant cet enthousiasme de lecture.

Dès sa première lettre envoyée le 3 février I9I4, Ritter reconnaît dans ce récit « un livre que je voudrais avoir fait et comme je ne saurai jamais en faire, hélas! ». C'est pourquoi il s'agira de défendre cette œuvre dans un compte rendu qui pourrait "donner le la à quelques confrères » et " faire honte à ceux qui crieront au scandale » (l.a.s. de Ritter à Carco, 23 février I9I4). Mais par la suite, il entretient aussi, progressivement, une sorte de « rêverie » amoureuse autour de son destinataire, malgré la distance géographique, l'éclatement de la guerre, les intervalles parfois très longs entre les réponses de Carco (il arrive que celui-ci ne réponde pas pendant plusieurs mois, au moment notamment de sa mobilisation). Les adresses de Ritter à son " soldat boulanger " seront tour à tour amicales, paternelles, affectueuses, séductrices, parfois marquées d'une irritation plus ou moins feinte : «Allons, mauvais enfant vicieux et charmant ; la voilà enfin votre petite lettre que je n'espérais plus ! (l.a.s. de Ritter à Carco, 9 mai I9I5). Carco est aussi l'objet d'une forme de projection imaginaire de la part de son correspondant, pour lequel il n'est pas loin, parfois, d'être une émanation de Jésus-la-Caille - ce qui le place dans la distance de la fiction : 
Encore une grande semaine passée, semble-t-il, et rien de mon soldat boulanger... Auriez-vous eu peut-être comme tant d'autres votre congé dont vous ayez, vous enfant terrible, usé et abusé. Avec qui ? Comment ? Vous seriez bien étonné si vous vous rendiez compte de quelle recrudescence de rêverie à ces choses votre cher livre est cause. Même, surtout, au milieu de la conflagration qui a tout emporté à vau l'eau des résistances et des volontés courageuses d'autrefois. / Je vous environne de ma rêverie lointaine :j'espère que vous ne le sentez pas trop, car je ne puis inspirer de réciprocité (l.a.s. de Ritter à Carco, 20 juin 1915).

La rencontre est toutefois, elle aussi, désirée, même si la différence des « milieux » et des habitudes sociales - comme la fréquentation des bars semble quelque peu redoutée par Ritter : "Quand nous rencontrerons-nous, cher ami, et nous rencontrerons-nous jamais? Pas dans un bar, toutefois. Si vous saviez quelle horreur instinctive j'ai de ces lieux. Et depuis l'enfance » (l.a.s. de Ritter à Carco, 9 mai I9I5).

Carco est d'ailleurs associé en pensée au petit groupe artistique et amical neuchâtelois que l'auteur d'Ægyptiacque fréquente depuis son retour de Munich, notamment à La Chaux-de-Fonds autour de Charles L'Eplattenier :

Sachez une fois pour toutes que notre vie quelle que ce soit n'est jamais sans penser à vous. Et même les oreilles ont dû vous tinter, ces dernières heures, car nous avons causé de vous à plusieurs reprises dans ce petit groupe de peintres et d'écrivains "avancés" de là-haut. Nous disons là-haut comme eux nous appellent $d u$ bas (l.a.s. de Ritter à Carco, 2I novembre I9I5).

Il souligne enfin l'intérêt de ses jeunes amis - Janko Cádra, CharlesÉdouard Jeanneret - pour cette œuvre. Le second possède désormais, dans sa bibliothèque, un exemplaire de Jésus-la-Caille - anecdote qui nous vaut un petit portrait assez curieux de l'architecte par son nouveau mentor :

Vous ne savez pas quelle sorte d'émotion j'ai eue à revoir la couverture jaune de la Caille dans la bibliothèque de Charles-Edouard Jeanneret. C'est l'architecte, aquarelliste hurluberlu et arrangeur d'appartements en « tableaux d'architecte ", comme disait hier la femme d'esprit chez qui nous soupions - [celui] dont je vous ferai faire quelque jour la connaissance à l'un de ses [bagnes ?] à Paris. Je crois vous en avoir déjà parlé. Il faudrait pourtant que je vous présente une fois par [la main ?] notre exceptionnel petit monde et tout d'abord, mon pluriel dont vous aurez peut-être autrefois parcouru les lettres tchèques au Mercure de France. L'intérêt pour vous de ce petit monde est très grand; je veux dire celui qu'il vous porte. Et ce sont braves et charmants garçons qui veulent que celà vous fasse plaisir. Quand nous serons les uns et les autres hors de nos misères on tâchera de se réunir au moins une ou deux fois l'an. Où que nous soyons (l.a.s. de Ritter à Carco, 2I novembre I9I5). 
Comme dans le cas du Corbusier, Ritter use aussi, bien entendu, de son statut d'aîné - il a vingt ans de plus que Carco - pour exercer son influence et donner des conseils'. Il l'encourage ainsi à écrire, à ne pas dilapider talent et énergie dans les bars et les mauvaises fréquentations. Surtout, il fait part de son inquiétude lorsque les réponses tardent, et qu'il imagine déjà Carco dans l'enfer des tranchées, grièvement blessé, voire tué :

Vous avez toujours tant aimé jouer avec le feu, enfant terrible, que j'ai peur ! / Si vous avez reçu le baptême du feu voici que subitement vous me semblez un être d'un autre monde. Si vous êtiez blessé il me semble que je le saurais. Si pire, que j'aurais eu un avertissement... peut-être reçu votre visite ! / C'est impressionant au possible d'écrire ainsi dans l'inconnu! (l.a.s. de Ritter à Carco, 22 octobre 1915).

Dans le même temps, ces conseils de sagesse et de tempérance se doublent, chez Ritter, d'une nostalgie de ses propres aventures de jeunesse, d'une liberté dont il aurait restreint, malgré lui, les possibles. Carco prend parfois à ses yeux la figure - sans doute fantasmée - d'un autre lui-même, à la fois envié et redouté ${ }^{10}$ :

Oh ! moi aussi j'ai beaucoup roulé ; mais je ne suis plus jeune. Moi aussi j’ai eu le goût des pires aventures : ma seule orginalité est peut-être d'avoir réagi. Mais je suis toujours celui qu'un certain chic canaille et le style chez le voyou peut enthousiasmer plus que tout au monde. Peintre je ne peindrais que cela. Ecrivain... [...] Si vous n'avez pas quitté Nouméa trop jeune, vous nous en donnerez j'espère le livre. Osez, osez, osez tout. J'ai été, moi, pris entre ma famille et mes scrupules religieux. Tout s'est résolu après tout le mieux du monde, puisque c'est fait et que je ne regrette rien. Mais vous, soyez donc ce que j'avais rêvé d'être. Si vous saviez de quel pas vainqueur j'étais parti... Et j'aurais eu de la joie à voir réalisé par vous à votre façon un peu des choses que j'avais rêvées, quelques uns des péchés que je me suis interdit de commettre et surtout les œuvres de volupté et d'amertumes qui pouvaient en résulter (l.a.s. de Ritter à Carco, Io février 19I4).

9 Ce qui ne l'empêche pas d'attendre en retour le jugement de son correspondant sur ses propres livres : «Je n'ai nulle foi en ce que je publie et seulement une peur horrible de vous raser et de vous paraître illisible. [...] Et je compte sur vous pour me dire la vérité féroce » (l.a.s. de Ritter à Carco, Io février I9I4).

Io Ritter transpose d'ailleurs sur cette relation avec Carco les rapports qu'il avait entretenus autrefois avec Pierre Loti (notamment lors de ses premiers séjours à Bucarest) : « [...] mon bel amour pour Pierre Loti ? qui lui aussi, alors, m'appelait "grand enfant"... Alors je vivais à Vienne avec Bojidar Karageorgevitch ; nous revenions de Dalmatie et d'Albanie ; alors... je devais singulièrement vous ressembler, Francis Carco... qui me ressemblez comme un petit frère... qui aurait mal tourné, mais qui a su écrire la Caille » (l.a.s. de Ritter à Carco, I2 juillet 1915). 
Dans ses lettres, Carco va se prêter d'assez bonne grâce aux « rêveries » et projections affectives dont il est l'objet, tout en mettant en évidence les différences qui le séparent de son correspondant. Il entretient d'abord l'ambiguïté du rapport épistolaire, qui oscille entre amitié et séduction amoureuse, et semble même rejouer - dans l'utilisation de formules oralisées - certains dialogues entre la Caille et ses amis : «Ah ! les putains ne manquent pas, mais en aurais-je envie ? Je n'ai envie de rien pour l'instant que de lettres d'amis sincères. Vous êtes un ami, s'pas ? » (l.a.s. de Carco à Ritter, 22 mai I9I5).

Surtout, il n'hésite pas à assumer le rôle du « mauvais garçon » - en grande partie parce qu'il lui permet de confirmer une « posture » à laquelle il s'attachera désormais : l'écrivain des «Apaches » et des mauvais lieux parlerait d'expérience et entretiendrait un rapport de proximité avec le monde décrit dans son œuvre ; par là, il y aurait une continuité entre la vie et le roman, dont l'authenticité se verrait ainsi confirmée:

Je suis né à Nouméa et j'ai pas mal couru la France, avec peu d'argent et le goût des pires aventures. Celles auxquelles je fais allusion dans Jésus-laCaille ne sont pas imaginées. Mais Montmartre dispense plus d'aventure que de joie (l.a.s. de Carco à Ritter, 8 février I9I4).

Cette expérience du « terrain » n'est d'ailleurs pas sans risque, et Carco se plaît à montrer qu'il éprouve dans sa chair les atteintes du monde interlope qu'il fréquente : «Je vadrouille et aujourd'hui je vous écris avec un œil poché comme un vulgaire voyou. C'est un souvenir de la nuit dernière, dans un bar. » (l.a.s. de Carco à Ritter, 23 mars I9I4). Il va même jusqu'à opérer une forme d'identification avec le métier exercé par les protagonistes de Jésus-la-Caille ; le romancier est en effet « une putain » : "J'étais plein d'argent, grâce à mon éditeur. J’ai tout dépensé... J’étais dans la joie... Ah ! vraiment, je ne suis qu'une putain et je me demande pourquoi j'écris. » (l.a.s. de Carco à Ritter, 25 janvier I9I6).

Une telle déconsidération de sa fonction auctoriale n'en demeure pas moins doublement équivoque. D'abord, elle sexualise sans doute à dessein sa position, dans un échange épistolaire fondé en partie sur une fantasmatique de l'« ambiguïté » de genre, dont Jésus-la-Caille est en quelque sorte le symbole ; ensuite, Carco semble conscient - et n'hésite pas, ici ou là, à le proclamer avec fierté - du caractère à la fois novateur et scandaleux (pour le «bourgeois ») de son œuvre, laquelle le vouerait à une réprobation sembable à celle dont sont victimes les « filles » et les « jésus » ${ }^{\mathrm{I}}$.

II Comme dans ce passage où Carco envisage une suite à Jésus-la-Caille : « Ah! vieille et terrible prostitution des quartiers neufs où la Caille se prendrait pour une jeune fille; masque affreux dont le jeune la Puce se pare pour faire l'amour avec les jolis garçons du Hasard ; or- 
Mais c'est bien la volonté de décrire des milieux en partie inexplorés, au potentiel romanesque et poétique intact, qui aiguise son ambition, et que Ritter ne cesse, pour sa part, d'encourager :

Je me sens capable d'un labeur immense sur des milieux de débauche et de crime qu'on connaît mal ou qu'on a vus avec un esprit bourgeois. Je suis trop curieux de toutes les débauches pour ne pas admettre ce que j'ai partout rencontré. Vive Zeus! cher ami... je renais à la vie. Je travaille et je vous aime de tout mon cœur! (l.a.s. de Carco à Ritter, 7 septembre 1915).

Pensez à mon enfance, aux longs voyages, à ma vie de demi-cabotin dans des bouis-bouis infects, de demi-apache dans les bals écartés des faubourgs, à mon amour de la crapule sensible. Oui, tout cela est neuf. C'est un monde boueux aux beaux yeux nostalgiques. Les fards et le beauté, le crime, le vol, la basse prostitution et les exotiques michés. Je ne suis pas un détraqué... mais je veux étreindre dans des livres ce grand corps tatoué d'une beauté inconnue. C'est un affreux désir qui pendant des années m’a jeté sur les pistes de toutes les grues de tous sexes. [...] Je n'ai qu'à me mettre devant une table avec beaucoup de papier blanc. Je n'aime pas les grande feuilles. Toutes ces bouches, maquillées ou non qui me furent « bonnes », chantent des paroles plaintives et misérables (l.a.s. de Carco à Ritter, 24 novembre 1915).

De manière symétrique, il s'agit aussi, pour Carco, de mettre à distance la «culture légitime » (au sens bourdieusien) : musique, peinture, littérature (la «tétérature », comme il la désigne dans sa lettre du Io décembre 1915) semblent susciter sa méfiance lorsqu'elles sont l'expression de la bourgeoisie bien-pensante ${ }^{12}$ comme des milieux « avancés ». Or, ce sont précisément à ces derniers que Ritter se pique d'appartenir, lui qui cherche une forme de distinction, dans un esprit antibourgeois très fin-de-siècle, en fréquentant l'aristocratie européenne (rappelons qu'il avait été, peu auparavant, lecteur de français de la princesse Rupprecht de Bavière, lors de son séjour à $\mathrm{Mu}-$ nich). Dans certaines lettres, il évoque avec la compétence qui est la sienne ses goûts picturaux ou musicaux, ce qui a sans doute suscité cette réaction de Carco :

gueil masculin et naïf de Bambou pour ses muscles (le chaste Bambou!). Je puis écrire un livre très serré de caractère... Mais qui ne me rejetera [sic], après, avec dégoût ? " (l.a.s. de Carco à Ritter, II mars I9I5).

I2 C'est ce que révèlent les attaques contre Barrès, par exemple, représentant-type de cette littérature "établie » et du « bourrage de crâne » durant la guerre : «Il y a dans les Innocents un ton de vérité qui sera durement attaqué et surtout une angoisse de tous les nerfs et un élan vers la pureté qu'on n'admettra pas. Mes crapules sont pourtant "vécues". Mais Barrès, mais les crétins du journalisme ! Barrès me dégoûte à la fin. Je ne puis vous écrire ce que j'ai appris de lui, de sa frousse en août dernier (I9I4). J'aurais peur qu'on n'arrête ma lettre. Il y a pourtant des façons toutes simples d'être un héros. Lui, fait trop de potin » (l.a.s. de Carco à Ritter, 3 novembre 1915). 
Pardonnez-moi de ne rien piger à la musique. Je suis un sauvage et mon plus grand bonheur est de m'étourdir dans d'infâmes guinguettes où guinchent les momacques de tout sexe... (l.a.s. de Carco à Ritter, 27 février I9I5).

De même, aucun goût particulier chez lui pour les livres, qui semblent, paradoxalement, avoir été contraires à sa vocation d'écrivain :

J'ai toujours voulu écrire, mais les livres m'étaient en horreur. Je m’empressais d'oublier ce qu'on m'apprenait. [...] Depuis l'oubli de toutes ces choses inutiles, le bienfaisant oubli m'a rempli la cervelle et m'a permis de m'étonner de la vie (l.a.s. de Carco à Ritter, Io décembre I9I5).

Ces propos relèvent, à n'en pas douter, d'une posture, puisque Carco a appartenu, dans les premières années de sa carrière, à un cercle de poètes - les «fantaisistes ", qu'il n'évoque d'ailleurs jamais dans ses lettres à Ritter pétris de tradition littéraire, de Villon à Verlaine, et conscients de la nécessité, propre à de nombreux courants de l'époque, de rompre avec la tour d'ivoire symboliste (dont ils ont, eux aussi, tâté comme apprentis poètes). L'admiration de Ritter (qui, par ailleurs, n'est peut-être pas si dupe de ce jeu de rôles) fournit l'occasion de mettre à l'épreuve cette nouvelle image auctoriale de « mauvais garçon » sans culture, attaché seulement à retranscrire toute vive son expérience - à la manière, précisément, du précédent de Villon ${ }^{13}$.

Certes, le "mauvais garçon » sait reconnaître l'apaisement bénéfique que lui procurent ces modèles qui lui sont antagonistes, constitués par la vie et l'œuvre de Ritter :

Heureusement que votre livre [Leurs lys et leurs roses ou L'Entêtement slovaque, dont Ritter a envoyé des exemplaires à Carco] si musicalement pur, si richement timbré d'émotion m'a sauvé quelques soirs des pires tentations. [...] Nous sommes très éloignés l'un de l'autre dans nos moyens d'expression. Pourtant, j'admire votre art si naturellement complexe et sinueux, votre goût du paysage, votre amour du développement (l.a.s. de Carco à Ritter, 22 avril I9I5).

Tout cela est assez honteux et je me dégoûte de vouloir exprimer la noblesse d'un monde dans lequel je n'aime que sa dépravation... Il faut attendre. Ça se tassera. Je suis intoxiqué. Mais je vous admire de vivre comme vous le faites dans cette petite chapelle où votre ami travaille près de vous ! J'envie votre sort... (l.a.s. de Carco à Ritter, 25 janvier I9I6).

I3 Figure clé de son panthéon personnel. Voir l'ouvrage qu'il écrira en 1932 sur le poète du Testament : Le Roman de François Villon. 
Carco va toutefois profiter de cet écart entre leurs moyens d'expression respectifs pour tenter de définir une sorte d'art poétique qui lui serait propre. Il le fait d'abord ici ou là, dans certaines lettres, comme en passant - et parfois en contradiction flagrante avec ce qu'il affirmera par ailleurs sur l'œuvre de Ritter : " Je ne cherche que le caractère sobre et violent des choses. L'expression trop étendue m'irrite et me rend injuste » (l.a.s. de Carco à Ritter, 23 mars 1914). Ce qu'il confirme encore, en mars I9I5, dans une comparaison fugace - mais révélatrice - avec les arts décoratifs :

J'aime la netteté dans tout, voire le cynisme... C'est une manie. Les objets de métal, la stricte splendeur d'un meuble... Le reste m'épaissit la vue et je le déteste. Non, le cahos [sic], l'expression touffue, l'abondance m'ont toujours étouffé. J'en reste aux formes pures, à la simplicité d'une ligne et c'est là toute ma vie... J'espère un jour en arriver à la traduction très dure et très sobre dans un livre colonial (l.a.s. de Carco à Ritter, II mars I915).

Mais ce sont surtout dans les deux longues lettres du 24 novembre et du Io décembre I9I5 qu'il se livre le plus explicitement sur ses conceptions de la littérature et du style. Il y exprime d'abord un refus « des idées, des principes ", une forme d'anti-intellectualisme. Encore une fois, seule compte pour l'écrivain la vie « vécue»:

Je ne sais pourquoi je vous écris ces folies, mais cela a été ma vie et, à présent, je regarde tous ces gens ["marlous", prostitué(e)es, etc.] qui ne me firent aucun mal et qui m'ont laissé vivre parmi eux comme un animal d'une autre race, et je veux les faire vivre dans mes livres tels que je les ai vus. Nos littérateurs se sont mis le doigt dans l'œil. Ils avaient des idées, des principes. Moi je n'avais rien. Tout me venait d'eux. J'avais la part du ciel et on ne s'occupait pas de mes caprices (l.a.s. de Carco à Ritter, 24 novembre 1915).

Il s'agit aussi, non sans provocation, de redonner sa place au succès - notamment financier - que l'on peut escompter des livres, et Carco ne cache pas, sur ce plan, ses ambitions, prenant ainsi le contrepied du principe d'autonomie propre aux milieux « avancés » de l'art pour l'art : « Je veux qu'on me foute la paix dans la vie avec les histoires idiotes des arrivistes. Je veux de l'argent pour mes livres et je serai toujours libre de faire ce qui me plait. C'est la vraie sagesse » (l.a.s. de Carco à Ritter, 24 novembre I9I5).

Il y réaffirme enfin la nécessité du style direct, sobre, non descriptif, au lyrisme tendu, en quelque sorte - et voit dans ce qui pourrait passer pour des lacunes auprès de ses confrères l'originalité de son œuvre à venir :

Il ne faut pas, cher ami, prendre trop au sériaux le type que je suis. Ça me donnerait des prétentions. Je suis un homme simple et qui n'a pas vieilli : 
une sorte de putain garçon. Et peut-être ne ferai-je rien de vraiment épatant? J'en ai parfois peur et parfois je me sens capable d'écrire des bouquins robustes qui étonneront tout le monde. C'est des idées, n'est-ce pas ? Et nous savons ce que valent les idées. Une bizarre destinée m'a toujours empêché de vivre dans les crises générales (littéraires ou militaires). Vous le voyez dans le cas présent. Et je m'en fous car j'ai un amour si malheureux de la beauté qu'il me console de ne pas faire figure parmi mes contemporains. On dit : Le petit Carco... Et un des mes copains m'appelle : Le fox-terrier... Bizarre ! Les mots me manquent : je suis très pauvre, un peu comme un sauvage qui fait son palais avec des matériaux qu'on dédaigne. D'où le pittoresque. Ah ! vraiment j'admire les littérateurs qui ont du lyrisme plein la gueule et plus d'expressions qu'ils n'en veulent. Alors, je vais droit aux objets. J'écris “il portait une casquette vieille" et je ne sais pas décrire cette casquette comme il est courant de le faire. Cette infériorité sur les confrères pourtant me ravit car elle me permet d'être exact (l.a.s. de Carco à Ritter, Io décembre I9I5).

Une comparaison avec le cubisme est aussi avancée, notamment avec le collage, que Carco infléchit dans son sens, avec une certaine ironie (pas sûr en effet que les peintres concernés se seraient reconnus dans une telle définition du procédé !), mais dont la fonction est sans doute de conférer une certaine radicalité moderniste à son dénuement stylistique :

Je comprends les cubistes qui, par défaut de moyens picturaux, collent sur leur toile un boîte d'allumettes véritable. Ainsi on ne se trompe pas. Mais je ne peux pas coller une boîte d'allumettes sur chacun de mes exemplaires. Alors je cherche à dessiner les objets dont j'ai besoin... et les confrères disent que je suis un roublard. [...] Ou je suis un âne ou j'ai quelque chose de remarquable à écrire (l.a.s. de Carco à Ritter, Io décembre 19I5).

Enfin, Carco avoue quel modèle implicite est le sien : c'est Maxime Gorki, dont il s'agit toutefois d'accommoder le réalisme à son propre tempérament :

Je voulais être quelque chose comme un Gorki français et je sens, maintenant, que je me rapproche de ce modèle. Mais il y a des différences de race. Pas de pitié chez moi. Une horreur de la laideur, du crime, des débauches vulgaires, une horreur et une attirance... Et je n'en sais pas plus long (l.a.s. de Carco à Ritter, Io décembre I9I5).

Ici s'arrête en effet notre parcours de l'art poétique de Carco, tel que celui-ci l'expose à Ritter.

Pour limitée qu'elle soit dans le temps et dans ses effets concrets pour les deux hommes, cette correspondance n'en est pas pour autant purement anecdotique, comme on a tenté de le montrer. Outre une amitié amoureuse que rien ne laissait prévoir, et qui semble plus marquée d'ailleurs du côté de Ritter, elle révèle les aspirations divergentes de ces écrivains au parcours 
et à la formation absolument dissemblables, et qui ont trouvé pourtant une plus-value dans cet échange. Ritter a sans doute découvert dans le récit de Carco - et dans ses lettres - une façon neuve de dire le désir et ses métamorphoses les moins acceptables aux yeux du public « bourgeois », franchise qui aurait pu servir d'exemple à ses propres tentatives romanesques. Il serait tentant de voir dans les récits plus tardifs et restés inédits, mais où l'homosexualité est davantage présente, une leçon de cette liberté revendiquée par le romancier-« Apache ». Carco présentait aussi, sans doute, une forme de modernité intégrable à l'esthétique de Ritter - modernité où la sobriété et la lisibilité du style, la poésie urbaine par touches discrètes, la « pudeur » du langage malgré le caractère "scabreux » du sujet, pouvaient convenir à un auteur très rétif à la radicalité des avant-gardes qui émergeaient alors, tout comme aux procédés, trop grossiers à ses yeux, du naturalisme.

Quant à Carco, c'est sans doute une oreille inattendue qu'il a trouvée tandis qu'il traversait une période de doute et de " cafard », liée bien sûr au contexte de la guerre, mais aussi à une phase particulière de sa carrière, où il renonçait à la poésie pour devenir romancier, selon un parcours typique de nombreux écrivains de l'époque (Ponton I975:67-68). Avec l'écrivain neuchâtelois, voici qu'il découvrait un admirateur attentif, mais à distance, loin du monde parisien, sur lequel éprouver la pertinence d'une image auctoriale renouvelée. Le "mauvais garçon » et l'esthète fin-de-siècle : c'est un peu le mariage de la carpe et du lapin - mais devenus chacun, par leur rencontre, plus conscients d'eux-mêmes et de leurs moyens respectifs.

\section{Bibliographie}

Barbey d'Aurevilly, Jules, Une vieille maîtresse - L'Ensorcelée - Un prêtre marié Les Diaboliques - Une page d'histoire, édition établie par Philippe Sellier, Paris, Robert Laffont, coll. «Bouquins », 20 IO.

Bedu, Jean-Jacques, Francis Carco au cœur de la bohème, préface de Pierre Bergé, Monaco, Éditions du Rocher, 200I.

Carco, Francis, Romans, édition établie et présentée par Jean-Jacques Bedu et Gilles Freyssinet, Paris, Robert Laffont, coll. « Bouquins », 2004.

Carco, Francis, Le Roman de François Villon, Paris, Éditions de La Table Ronde, 1996.

Décaudin, Michel, Les Poètes fantaisistes : anthologie, Paris, Seghers, coll. «P.S. », 1982.

Ponton, Rémy, « Naissance du roman psychologique. Capital culturel, capital social et stratégie littéraire à la fin du XIX ${ }^{\mathrm{e}}$ siècle ", Actes de la recherches en sciences sociales, $\mathrm{n}^{\circ} 4$, juillet I975, pp. 66-8I. 
Ritter, William, « Journal » (I2 août I9I3 - 22 janvier I9I4 et 22 janvier I9I4 - 3 juillet 19I4, grand cahier noir, Munich), Fonds Ritter, Archives littéraires suisses, Berne. 